\title{
Correction to: Appraisal of disease-modifying potential of amlodipine as an anti-arthritic agent: new indication for an old drug
}

\author{
Sumera Qasim ${ }^{1,3} \cdot$ Alamgeer $^{1,2}$ (D) Saima Kalsoom ${ }^{4} \cdot$ Muhammad Shahzad $^{5} \cdot$ Hafiz Muhammad Irfan $^{1}$. \\ Muhammad Shoaib Zafar ${ }^{5}$. Ishfaq Ali Bukhari ${ }^{6} \cdot$ Fahim Vohra $^{7}$. Sibtain Afzal ${ }^{8}$
}

Published online: 2 May 2020

(c) Springer Nature Switzerland AG 2020

\section{Correction to: Infammopharmacology https://doi.org/10.1007/s10787-020-00692-9}

Unfortunately, the 4th author name was incorrectly published in the original publication. The complete correct name is given below.

Muhammad Shahzad.

The original article has been updated.
Publisher's Note Springer Nature remains neutral with regard to jurisdictional claims in published maps and institutional affiliations.

The original article can be found online at https://doi.org/10.1007/ s10787-020-00692-9.

Alamgeer

alam_yuchi@yahoo.com

1 Laboratory of Cardiovascular Research and Integrative Pharmacology, College of Pharmacy, University of Sargodha, Sargodha, Pakistan

2 Punjab University College of Pharmacy, University of the Punjab Lahore, Lahore, Pakistan

3 College of Pharmacy, Jouf University, Sakaka, Al Jouf, Saudi Arabia

4 SA-CIRBS International Islamic University Islamabad Pakistan, Islamabad, Pakistan

5 Department of Pharmacology, University of Health Sciences, Lahore 54600, Pakistan

6 Department of Pharmacology, College of Medicine, King Saud University Riyadh, Riyadh, Saudi Arabia

7 Department of Prosthetic Dental Sciences, College of Dentistry, King Saud University, Riyadh, Saudi Arabia

8 Immunology Research Center, College of Medicine, King Saud University Riyadh, Riyadh, Saudi Arabia 